\title{
EMPRESAS VERSUS MERCADOS. UN BOCETO PARA UNA HISTORIA DE LA EMPRESA $\left(1 .^{a}\right.$ parte $) *$
}

\author{
SEBASTIAN COLL MARTIN \\ Universidad de Cantabria
}

Casi por definición, las vísperas de un proceso de diseño de planes de estudio constituyen el momento oportuno para reflexionar sobre la procedencia de introducir en la enseñanza una nueva materia, y para plantearse la pregunta - indisolublemente unida a la anterior- del contenido que se quiere dar a esa materia.

¿Merece la pena que los historiadores económicos propongamos una asignatura de historia de la empresa en la licenciatura de empresariales o en la de económicas? $\mathrm{O}$, planteado de otra forma, conocer el desarrollo histórico de la empresa, ies para un licenciado en empresariales o en económicas lo suficientemente útil como para reservar a este fin un hueco en los planes de estudio, habida cuenta de que el tiempo es para el estudiante un recurso escaso?

En las páginas que siguen se da una respuesta positiva a esta pregunta, aunque matizada por una serie de condiciones. En efecto, la historia de la empresa, como cualquier historia, puede ser algo perfectamente inútil o, por el contrario, sumamente útil: todo depende de que seamos capaces de convertirla en algo así como "lo que usted quería saber acerca de la empresa, y no le cuentan en otras asignaturas». Naturalmente, me apresuro a pedir disculpas a los que imparten esas otras asignaturas, y a asegurarles que ese supuesto descuido no les es imputable a ellos, sino a la división del trabajo, que - sigo suponiendo- dificulta el que alguien se ocupe de las cuestiones más generales.

Una de las cosas que se necesitan para convertir a la historia de la empresa en eso que, sin ninguna originalidad, estoy proponiendo es que nosotros mismos nos hagamos aquellas preguntas relevantes que, por unas

\footnotetext{
* Una versión anterior de este trabajo fue presentada al Primer Seminario de Historia de la Empresa, celebrado en Granada los días 10 y 11 de enero de 1991. Agradezco a los participantes en el Seminario así como a su organizador, Gregorio Núñez, los comentarios efectuados. También agradezco sus comentarios a un anónimo lector árbitro, que señaló algunas deficiencias del artículo. De aquellas que han sobrevivido, me declaro único responsable.
} 
razones o por otras, estén descuidadas en otras materias. La ventaja comparativa de los historiadores está en el largo plazo, que es tanto como decir en la ausencia de preocupación por lo inmediato. Por ello, como los adolescentes que no tienen que preocuparse por la comida o el alojamiento, estamos en las mejores condiciones para plantearnos cuestiones profundas y trascendentes del tipo de las siguientes:

- ¿qué es en realidad la empresa?;

- ¿por qué la empresa?;

- ¿cuáles son los determinantes del tamaño y forma de la empresa?;

- ¿y cuáles son las tendencias a largo plazo en este último terreno?

Desde luego, estas preguntas no son nuevas, y con tanto derecho como los historiadores se las pueden plantear $-y$ se las han planteado de hecho- los economistas teóricos. El riesgo que corren, y me temo que su destino en muchos casos, es el de encontrar más audiencia entre los historiadores que entre los mismos economistas. Siempre he creído en los beneficios de la colaboración entre teoría e historia económicas - lo cual tampoco tiene nada de original-, y por ello, como muchos otros historiadores económicos, creo que nuestras hipótesis de trabajo deben inspirarse en los trabajos de indole teórica. En atención a ello, permítaseme empezar con un repaso no exhaustivo de algo que podríamos llamar la high theory de la empresa.

\section{UN POCO DE TEORIA DE LA EMPRESA}

Una definición de la empresa nos dirá que ésta es el lugar en el que se asignan los factores de producción para la obtención de bienes. Sin embargo, eso mismo es lo que, según los manuales de teoría económica, hacen el mercado y el mecanismo de los precios. También podemos leer que la empresa nace de la división del trabajo, y uno no tiene más que recordar la descripción de la fábrica de alfileres de Adam Smith, o la "Fábula de las abejas" de Bernard de Mandeville, para obtener una brillante ilustración de una afirmación como ésta. Unicamente cabe objetar que, en los mismos autores, el mercado aparece también como un resultado de la división del trabajo.

El mercado es, pues, un lugar de encuentro entre agentes económicos -empresas con consumidores, o empresas entre sí-, y provee a éstos de unas señales - los precios - en las cuales basar su actuación. La empresa es, asimismo, un lugar de encuentro entre agentes económicos, cuya actuación dirige dictándoles órdenes. 
Quizá lo más sorprendente de todo es que una misma transacción, por ejemplo la adquisición de un input intermedio por parte de una empresa, en unos casos se confía al mercado, mientras que en otros casos es interna a la empresa, que toma a su cargo la producción del mismo, La pregunta siguiente es, por tanto, la de qué determina que una transacción concreta sea interna a la empresa o externa a ella, o, si se quiere, por qué los obreros de la fábrica de alfileres de Smith formaban una única empresa en lugar de venderse los alfileres a medio procesar, como producto intermedio, los unos a los otros. Esta pregunta es tanto más relevante por cuanto que la sustitución del mercado por la empresa, de la mano invisible del mercado por la mano visible del management, para utilizar la feliz expresión de Alfred Chandler ${ }^{1}$, parece constituir un fenómeno progresivo, algo así como una tendencia histórica.

Espero que a estas alturas, como decía Ronald Coase, «el lector aceptará como rasgo distintivo de la empresa su función de sustituir al mecanismo de los precios" ${ }^{2}$, y espero también que mi escasa elocuencia al tratar de transmitir lo que otros mejores han dicho antes, no habrá oscurecido del todo la relevancia de las preguntas planteadas y el alcance de sus implicaciones.

Empecemos, pues, por la cuestión de la esencia de la empresa. Si tratamos de encontrar una definición funcional de la misma que no nos sirva también como definición de lo que hace el mercado, esto es, si definimos la empresa por oposición al mercado, resaltaremos sus siguientes rasgos distintivos:

- En la medida en que las partes dentro de una empresa están ligadas por una relación contractural, esos contratos tienen por objeto a los factores, no a los bienes; estos últimos pasan físicamente por varias manos en el curso del proceso productivo, sin que su propiedad se transmita de forma paralela.

- La relación entre las partes que nace de esos contratos aparece, por lo demás, como una relación jerárquica: lo que una de las partes obtiene en virtud de tales contratos es el derecho a disponer del recurso de que originalmente es propietaria la otra parte.

- Finalmente, la relación es también desigual en otro sentido, en el de la distribución del ingreso: mientras que el empresario obtiene una retribución variable, ligada a la productividad de los factores, éstos obtienen una retribución fija ${ }^{3}$.

1 Chandler (1977).

2 Coase (1980), p. 68.

3 Por supuesto, la teoría económica nos dice que también esa retribución fija está ligada a la productividad de los factores, a través de la igualdad entre el precio de un factor y el valor de su producto marginal. Sin embargo, y haciendo por ahora abstracción de otras 
El por qué de todo ello es una pregunta que ha recibido respuestas diferentes a lo largo del tiempo. Algunas de ellas serían las siguientes:

- En primer lugar, si se efectúa una asimilación del tipo empresario igual a propietario del capital y factor contratado igual a trabajo, y si existe una escasez relativa del capital con respecto al trabajo -o acaso de los capitalistas frente a los trabajadores-, puede intentarse una explicación de la desigualdad distributiva inherente a la empresa análoga a la que Ricardo construyó para ilustrar la desigualdad retributiva que surge en el mercado de tierra. Supongo que eso es lo que se quiere significar cuando se utiliza el término "capitalismo".

Los inconvenientes de esta explicación son, sin embargo, varios. En primer lugar, la asimilación de un recurso producible por el hombre - el capital- con uno no reproducible - la tierra - puede resultar abusiva, y de hecho en aquella parte de la teoría económica que trata de los mercados de factores no se encuentra nada que avale la legitimidad de esa analogía. Por lo demás, en el análisis de Ricardo, la parte que a largo plazo se lleva la totalidad del excedente no asume ni la decisión ni el riesgo - o el beneficio en el corto plazo-, en contra de lo que sucede en el interior de la empresa.

En segundo lugar, la asimilación del empresario con el propietario del capital parece también abusiva, toda vez que los empresarios actúan de forma habitual como demandantes de capital, y los propietarios de este último adoptan una posición subordinada similar a la de los trabajadores.

- Se ha argumentado que la primera de estas dos objeciones conserva su validez para el caso de que, a la vista de la segunda, adoptemos la postura - similar - de considerar que el recurso realmente escaso es la capacidad empresarial. Este era el núcleo de la explicación de la empresa que daba Frank Knight en la primera parte del presente siglo 4 , una explicación que en parte apelaba a los costes de la información y, en parte, a variables psicológicas. En efecto, para Knight, en este mundo hay algunas personas bien informadas, decididas y seguras de sí mismas, en tanto que otras - ila mayoría? - están mal informadas, son indecisas y son inseguras. Los primeros serían los empresarios, y los demás los que ceden los factores de su propiedad a cambio de una retribución cierta.

Coase criticó en su día que esta explicación de Knight no deja claro por

cuestiones, eso significa ni más ni menos que una relación entre la retribución de las sucesivas unidades de un factor $\mathrm{X}$ y la productividad de la unidad iésima de ese factor $\mathrm{X}$. Lo que se puede uno cuestionar $-\mathrm{y}$ lo que parecen haberse cuestionado los trabajadores a lo largo por lo menos del último siglo- es la necesidad de que sea precisamente la productividad de la última unidad la que dicte el salario de todos ellos.

4 Knight (1921). 
qué los bien informados no venden simplemente su información, como de hecho hacen a diario muchos profesionales independientes del sector servicios, en lugar de echarse sobre las espaldas las tareas de coordinación. Simultáneamente, a Coase no le queda claro por qué la retribución cierta que obtienen las otras partes ha de ir unida a la pérdida del poder de decisión, y encuentra que la relación inevitable entre ambas cosas queda desmentida por la cantidad de contratos de obra que se firman a diario ${ }^{5}$.

- En 1937, Ronald Coase publicó un artículo justamente famoso que llevaba precisamente por título el de "La naturaleza de la empresa", y que se ha convertido en el punto de partida de casi toda la literatura posterior en torno a esta temática. Para él, la clave de la sustitución del mercado por la empresa en una serie de funciones relacionadas con la asignación reside en que la utilización del mercado implica costes, costes de transacción. Más en concreto, identifica los siguientes:

En primer término, costes de información. En palabras de Coase, "el coste más obvio de "organizar" la producción a través del mecanismo de los precios es el de descubrir cuáles son los precios relevantes" ${ }^{6}$.

En segundo lugar, los propios costes de negociar. Toda negociación consume recursos, y alguien - quizá el propio Coase-enunció un teorema que dice básicamente que los costes de transacción aumentan - no recuerdo en qué progresión- con el número de los participantes en ella.

Una implicación central de todo esto - no la única - es que, en una transacción que envuelva a numerosas partes, se ahorran costes si una de ellas adopta una posición central y ultima acuerdos bilaterales con cada una de las demás partes, en lugar de perseguir un único acuerdo multilateral o una miríada de acuerdos bilaterales entre todos y cada uno de los afectados.

Otra implicación asimismo señalada por Coase de los costes de negociación es que, ceteris paribus, resulta económico sustituir contratos a corto plazo por contratos a largo plazo, aunque para llegar a un contrato a largo plazo haya que dejar inespecificados muchos detalles: esa falta de especificación se supliría por una cláusula en virtud de la cual una de las partes decidiría discrecionalmente.

5 La posición de Knight sigue siendo defendible, sin embargo, desde varios puntos de vista. Por ejemplo, si en lugar de fijarnos en la información consideramos que el recurso verdaderamente escaso es precisamente la capacidad de organizar y decidir. McManus (1975) hace una reivindicación de Knight parecida a ésta, al considerar que lo que caracteriza al empresario de Knight es la posesión simultánea de dos especialidades: la afición por el riesgo y la capacidad de organizar.

6. Coase (1980), p. 69. Para un análisis más detallado de los costes de transacción, las formas de gobernar transacciones y los consiguientes modelos de organizaciones, puede verse Salas (1984). 
Por último, cuanto más versátil sea el objeto de la negociación, más detalles quedarían sin especificar en un contrato: por ello, siempre según Coase, los contratos que tienen por objeto a los factores dejan más a la discreción de una de las partes - son más asimétricos- que los contratos que toman por objeto a los bienes.

- En un trabajo bastante posterior en el tiempo, Armen Alchian y Harold Demsetz ${ }^{7}$ han argumentado que, si bien Coase tenía razón al aludir a los costes de transacción y había conseguido explicar bien en base a ellos la posición central de una parte dentro de la empresa, en cambio, no había tenido el mismo éxito a la hora de desentrañar por qué esa parte central tiene que desempeñar una función de control y percibir una retribución variable, dos rasgos que caracterizan en la práctica al empresario.

Para llegar a esa explicación, Alchian y Demsetz partían de los costes de información, que Coase había identificado como costes de descubrir "los precios relevantes" de los inputs integrados en la empresa. Lo que caracteriza a la empresa es, para Alchian y Demsetz, la producción en equipo. E1 output obtenido por un equipo es indivisible, en el sentido de que los distintos miembros son físicamente incapaces de obtenerlo cuando actúan por separado - pensemos en una función de producción capital y trabajo, o en el maquinista y el fogonero en un ingenio de vapor-, y las cosas no cambian mucho si en lugar de ello admitimos que los miembros del equipo, actuando por separado, pueden también producir un output, pero significativamente menor que aquel que obtienen conjuntamente. Supuesto que, por alguna de estas dos razones, el equipo existe, la indivisibilidad de facto de su output plantea el problema de determinar cuál es la contribución al mismo de cada miembro. Planteadas así las cosas, la determinación de la contribución de los factores y, consiguientemente, el reparto del ingreso entre esos factores, la determinación de sus precios, se convierte en una cuestión insoluble.

Si, por un lado, resulta imposible determinar la contribución de cada miembro del equipo al resultado final, por otro lado, no cabe duda de que la productividad de dichos miembros será mayor o menor dependiendo de lo mejor o peor que éstos apliquen sus facultades. Naturalmente, cada miembro del equipo tiene su mapa de curvas de indiferencia que relacionan ocio e ingreso. Si el individuo, trabajando en equipo, opta por haraganear, la indivisibilidad del output implica que sólo tecaerá sobre él una pequeña parte de la reducción consiguiente del ingreso. Eso significa que en todo equipo existe un incentivo para haraganear; a continuación, como todos los miembros del equipo experimentan el mismo incentivo, el resultado será

\footnotetext{
7 Alchian y Demsetz. (1972).
} 
demasiado ocio y poco trabajo, alejando a los individuos de la combinación que maximiza su utilidad, de la combinación que elegirían trabajando en solitario. El parentesco con algunas de las teorías de la hacienda pública y con la teoría económica de los derechos de propiedad - en la que Demsetz y Alchian ocupan lugares destacados- es fácil de establecer.

En unas condiciones como éstas, parece necesario encontrar otro sistema de remuneración de los factores - una retribución básicamente fija-y algún sistema corrector que eleve el esfuerzo productivo de los miembros del equipo en su propio beneficio; sin embargo, por principio, ese sistema corrector no podrá basarse en una medición de la (inobservable) productividad de cada miembro. El resultado es que habrá que recurrir a una medición indirecta de la productividad de cada miembro del equipo, observando su comportamiento. En eso consistiría la tarea del controlador. La conclusión de Alchian y Demsetz es, pues, que los miembros del equipo están interesados en la presencia de un control y de un controlador, por supuesto siempre que los costes que éstos implican sean inferiores a los costes que evitan.

¿Y quién controla a los controladores, esto es, cómo se garantiza que ellos mismos se aplican en su tarea? A esta pregunta, Alchian y Demsetz contestan que a los controladores no se les controla; se les estimula. El estímulo consistiría en ligar su retribución a la productividad del equipo, para lo cual se les reserva el resto que queda del ingreso una vez que los demás miembros han percibido su retribución. Es por ello que la función de control va unida a una retribución variable.

El controlador de Alchian y Demsetz se caracteriza, pues, por su posición central en el entramado de contratos que ligan a los miembros de la empresa, por su poder de asignar tareas a los miembros del equipo y de controlar su comportamiento, por el poder de alterar la propia composición del equipo, por su retribución variable $y$, por último, por la propia capacidad de vender todos estos derechos. Parte de esos poderes son lo que corrientemente se define como propiedad, y el controlador de Alchian y Demsetz quedaría investido, en definitiva, de los rasgos que caracterizan a la figura del empresario clásico ${ }^{8}$. Dejamos para más adelante una referencia a las causas y consecuencias de la cada vez más frecuente segregación de la propiedad y el control, la cual constituye el tema de la teoría de la agencia.

Finalmente, se puede añadir que Alchian y Demsetz discuten la noción de Coase del carácter jerárquico de las relaciones en el seno de la empresa, a cuyo efecto apuntan que el único poder coercitivo real de que dispone el empresario es el de rescindir el contrato, despidiendo a los miembros, posi-

8 En esta descripción, la empresa aparece, por tanto, como una forma de contrato social. Seguramente es ocioso añadir que esta explicación no pretende seguramente reconstruir un proceso histórico; una interpretación alternativa sería la de que esta solución, más eficiente que otras alternativas, se impone en la práctica a cllas. 
bilidad que también poseen estos últimos ${ }^{9}$. Por lo demás, no resulta superfluo recordar que, pese a sus disensiones con respecto a Coase, se encuentran en la estela de este último, analizando uno de los costes de transacción identificados por él: los costes de medición de determinados inputs.

- En otro interesante estudio acerca de la naturaleza de la empresa, debido a John McManus ${ }^{10}$, los costes de medición de los inputs ocupan también un lugar central. McManus no se ocupa, como Alchian y Demsetz, del problema de la indivisibilidad del output y de la imposibilidad en ciertos casos de llegar a una medición directa de la contribución de un factor. Lo que señala McManus es, más bien, que todo objeto de transacción ha de ser, para llegar a ésta, contado, pesado y medido, y que a menudo esa medición debe extenderse a cualidades difíciles de comprobar: pensemos en el sabor de los alimentos, lo que nos va a durar una prenda de vestir, o la probabilidad de avería y la subsiguiente facilidad de reparación de una máquina. Es por ello que nuestras mediciones son a menudo muy imperfectas, y que debemos recurrir a sustitutos tales como controles de calidad, inspecciones, normalización, marcas y etiquetas.

Tanto la medición propiamente dicha como sus sustitutos comportan costes, que en ciertos casos pueden llegar a ser muy altos. McManus compara esos costes, que se incorporan al precio de venta, con un impuesto indirecto que perjudica a productores y a consumidores y que, por tanto, tiene un efecto negativo sobre el bienestar social. Son tales costes lo que tratarían de evitar las empresas internalizando, o hurtando al mercado, una parte de las transacciones. En concreto, aquellas para las cuales el control interno constituya una alternativa más barata.

- No todos los trabajos que interpretan a la empresa como un resultado de los costes de transacción están centrados, como los dos reseñados en último lugar, en los costes de medición de los inputs. El propio Alchian, en unión esta vez de Klein y Crawford, e inspirándose probablemente en trabajos previos de Oliver Williamson y de Victor Goldberg ${ }^{11}$, analizó otro tipo de costes de transacción, el coste de hacer cumplir los contratos o el riesgo de un comportamiento oportunista postcontractual ${ }^{12}$.

Lo que hace potencialmente importantes a estos costes de un comportamiento oportunista postcontractual es la existencia de cuasi rentas en una

- También apuntaban que, en condiciones de pleno empleo - la fecha de su artículo es 1972 - La efectividad de esta sanción no es mayor que la que pueda tener el "despido" de un tendero por parte de un cliente.

10 McManus (1975).

11 Véanse Williamson (1971) y (1975); Goldberg (1976).

12 Klein, Crawford y Alchian (1978). 
economía, y la relación de éstas con grandes inversiones. Un ejemplo de todo esto a pequeña escala podría ser el siguiente:

Supóngase que este trabajo fue pasado a máquina por una mecanógrafa, y supóngase, asimismo, que el mercado en el que ésta y el autor contactaron era un mercado notablemente imperfecto. Imaginemos, por ejemplo, que había escasez de mecanógrafas, y que el autor aceptó pagar a una de ellas un total de 100.000 pesetas por la tarea. Imaginemos también que los clientes de las mecanógrafas son asimismo raros en ese mercado, hasta el punto de que, en ausencia del autor, la chica contratada hubiera tenido que emplearse en un trabajo diferente al suyo, cobrando, digamos, sólo 50.000 pesetas. En estas condiciones, la cuasi renta que obtendría por poseer una habilidad - un activo- escasa se cifra en 50.000 pesetas (=100.000-50.000). Naturalmente, la mecanógrafa aceptaría trabajar también por cualquier otra suma que excediese al coste de oportunidad de su tiempo de trabajo, esto es, mientras obtenga una cuasi renta positiva.

Una consecuencia de ello es que, si las circunstancias desde el punto de vista legal se lo permiten, el autor, una vez establecido el acuerdo, podrá rebajar unilateralmente la cantidad pagada, y la mecanógrafa seguirá trabajando para él siempre que el nuevo precio le siga dejando una cuasi renta. Incluso, si la rebaja tiene lugar una vez becho el trabajo - esto es, la inversión-, el autor podrá teóricamente obtener las hojas mecanografiadas por cualquier precio simplemente superior a cero, toda vez que, para la mecanógrafa, esas hojas tienen una utilidad cero.

Incluso en el caso de que mediase un contrato escrito, hay bastantes probabilidades de que ese oportunismo postcontractual quedase impune: por ejemplo, si mi mecanógrafa considera que son demasiado elevados los costes de acudir a la justicia, si es incierto para ella el fallo de esta última, si por alguna razón no especificada hasta ahora la chica experimenta una pérdida importante por interrumpir su trabajo, etc.

En el mundo real, los mercados son, con bastante frecuencia, imperfectos, sobre todo cuando uno se mueve al nivel de grandes contratos, y existen por ello cuasi rentas importantes; también las inversiones que genera la firma de un contrato pueden llegar a ser grandes, las interrupciones en la producción salen caras, y en general se dan a menudo las condiciones para el oportunismo postcontractual. Klein, Crawford y Alchian consideran que estos costes son los que están detrás de la decisión de muchas empresas de convertirse en su propio proveedor o en su propio cliente, poniendo en marcha procesos de integración vertical.

Lo que hemos resumido en las páginas anteriores no es, desde luego, todo lo que se ha escrito sobre el tema de la naturaleza de la empresa ${ }^{13}$,

\footnotetext{
13 Para completar esta visión, véase, por ejemplo, Salas (1984).
} 
pero seguramente es suficiente para dar una idea del estado de la cuestión: hay una tendencia a considerar en el origen de ésta a los costes de transacción, que se identificarían principalmente como costes de informaciónmedición, y costes motivados por el oportunismo postcontractual; quizá se pudiera añadir un tercer coste inherente a la propia negociación, en aquellos casos en los cuales no hay un precio establecido por un mercado impersonal, sino más bien un proceso del tipo caja de Edgeworth.

La siguiente cuestión teórica es la relativa al tamaño y características de la empresa.

- Quizá no esté de más empezar con una referencia a la teoría de la oferta, y a su predicción de que la empresa productora de un único bien llevará la producción de éste, y por tanto crecerá ella misma, hasta aquel punto en el que se igualen el coste y el ingreso marginal. La insuficiencia de este enfoque reside, por un lado, en que son relativamente pocas las empresas que producen un único bien, y por otro lado en que no se plantea la cuestión de si el proceso de producción de ese bien es fraccionable -o está fraccionado de hecho- en varios subprocesos, cada uno de los cuales puede ser -o es- protagonizado por una empresa diferente. En otras palabras, si bien la teoría de la oferta nos dice cuál será la dimensión, por así decirlo, horizontal de la empresa, en cambio, tal teoría parece mal equipada para explicar el grado de integración vertical de la misma.

- Es vieja la proposición según la cual el tamaño de una empresa tiene un límite superior impuesto por los rendimientos decrecientes a la dirección. La incapacidad de la dirección para encontrar la combinación más eficiente de los factores a partir de un determinado volumen de los mismos podría proporcionar una respuesta a la pregunta acerca del tamaño de la empresa. Tal respuesta, que en su día dieron, entre otros, Nicholas Kaldor y E. A. G. Robinson ${ }^{14}$, tiene el atractivo de que no se refiere sólo a la dimensión de la empresa en horizontal, pero, en cambio, presenta el inconveniente de resultar demasiado poco precisa.

- Seguramente, también es incompleta, ya que se fija sólo en cómo evolucionan los costes de control de la empresa, sin aludir a las diferencias existentes entre distintas empresas o distintos inputs, y sin aludir tampoco a los costes de la organización alternativa a la empresa, el mercado. Por ello, el artículo de Coase, que empezaba preguntándose por estos últimos, proporciona una visión más global. Coase aceptaba el carácter creciente de los costes de dirección, y llegaba a la proposición de que la empresa internali-

14 Kaldor (1934); Robinson (1934). 
zaría sus transacciones hasta aquel punto en el cual el coste de hacer una transacción más en el seno de la empresa se igualase con el coste de efectuarla en el mercado.

Como proposiciones derivadas de ésta predecía, en primer lugar, que la tendencia al crecimiento de una empresa concreta será tanto mayor cuanto menores sean sus costes de organización, y cuanto menos crezcan al aumentar el número de transacciones. En segundo lugar, deducía que todas aquellas innovaciones que facilitasen la comunicación - reduciendo con ello los problemas de la dispersión espacial aneja al crecimiento de la empresaredundarian, ceteris paribus, en un aumento del tamaño de esta última.

- Alchian y Demsetz, con su insistencia en el carácter indivisible del output de la empresa, parecen apartarse poco de la teoría estándar de la oferta, que explícitamente supone la homogeneidad del output e implícitamente la indivisibilidad del proceso de producción. Sin embargo, acto seguido admitieron un caso en el cual el equipo y su output resultasen divisibles, pero al precio de una reducción de este último.

Con ello, el tamaño de la empresa vendría determinado por la comparación de los costes inherentes a la fragmentación del equipo con los costes en los que se incurre al implantar y extender el mecanismo de control. Básicamente, la condición de equilibrio vendría a ser así la misma que en Coase. La novedad consiste en su matización de que el coste de observar desde fuera el comportamiento de un miembro del equipo es diferente según cuáles sean las características de éste, lo cual constituye un punto de partida para llegar a predicciones algo más concretas.

Tanto o más que al tamaño de la empresa, esas predicciones de Alchian y Demsetz se referían a la forma de organización de ésta. Así, afirmaban que, toda vez que en el trabajo físico o mecánico es más fácil encontrar indicadores externos de la productividad que en el caso del trabajo intelectual o especializado, el control externo sería más fácil en el primer caso; por ello, y a diferencia de lo que sucede en las fábricas, en los despachos de los abogados, gabinetes médicos, colectivos de artistas y otras empresas similares, no existirían supervisores, y el control se vería sustituido por el incentivo, en forma de participación en los beneficios. Si los individuos son pocos y se conocen entre sí, el resultado será la sociedad de pérdidas y ganancias.

Cuando las necesidades de capital de la empresa hacen necesaria la sociedad anónima, entonces el controlador deja de ser el propietario, o al menos el único propietario. Puesto que ahora no se le puede estimular retribuyéndole con el resto íntegro del ingreso, una vez deducida la retribución (fija) de los otros factores, se hace necesario controlar al controlador, para lo cual nacería el consejo de administración. 
Ottas predicciones de Alchian y Demsetz hacen referencia a las necesidades de control en la empresa socialista, y una particularmente atrayente - por cuanto parece confirmada en la realidad- dice que las mutuas de seguros y los clubs deportivos son ineficientes casi por definición. Asimismo, llegan a proposiciones acerca de la propiedad de las herramientas usadas por el trabajador - si suyas o de la empresa-, basándose en la mayor o menor dificultad de controlar el uso que hace de ellas: así, cuando es un único individuo el que emplea la herramienta, o cuando el propietario es absentista, las herramientas tienden a ser propiedad del trabajador.

- Quizá el punto más débil de la argumentación de Alchian y Demsetz consista en su suposición implícita de que una sociedad anónima es capaz de controlar eficientemente a los gerentes, sin entrar en los mecanismos de ese control y en los conflictos que le hacen necesario. De estas cuestiones se ocupa la llamada teoría de la agencia, desarrollada, entre otros, por Arrow, Fama, Ross, Jensen y Meckling, y que ha dado lugar a una pléyade de trabajos. Estos, de naturaleza teórica unos y empírica otros, se centran en dos cuestiones principales. Por un lado, el funcionamiento del control externo que supone la existencia de un mercado laboral de directivos exterior a la empresa y que liga los sueldos de éstos a los resultados de su gestión anterior. Por otro lado, la eficiencia del control interno que pueda ejercer el consejo de administración, tema que remite al de su composición ideal, entre propietarios del capital que a menudo no poseen la capacidad para ejercer esa labor, los propios managers susceptibles de competir entre sí por la gerencia, y los consejeros externos a la empresa, cuando exista el riesgo de que esa competencia se convierta en colusión.

- La aportación de McManus, centrada en los costes de medición de los inputs - cantidad y calidad-con independencia de que el output sea o no divisible, hace depender el tamaño de la empresa de la dificultad de esa medición. Igualmente avanza la proposición de que el factor que resulte más difícil de medir pasará a ocupar la posición central en la empresa, esto es, pasará a convertirse en el empresario.

Así, por ejemplo, en la agricultura, dependiendo de qué input sea más difícil de medir en un momento dado, la relación entre propietarios de la tierra y propietarios del trabajo dará lugar a:

- el arrendamiento (cuando la calidad del trabajo se convierta en una cuestión central);

- la explotación directa por el propietario con trabajo asalariado (cuando la diversidad de calidades de la tierra haga difícil su medición); 
- o la aparcería, cuando la dificultad de medir la calidad de ambos inputs resulte similar.

- Por su parte, las predicciones de Klein, Crawford y Alchian, centrados como están en el problema del oportunismo postcontractual en los mercados de bienes, se refieren fundamentalmente al grado de integración vertical de las empresas. Para ellos, la integración vertical dependerá:

- de la importancia de las cuasi rentas, las cuales, a su vez, aparecen relacionadas con el volumen de las inversiones de las empresas en activos fijos o en capital humano;

- de lo costoso que resulte para las empresas el tiempo necesario para encontrar clientes o proveedores alternativos ${ }^{15}$;

- de lo costoso que resulte hacer cumplir los contratos por medios legales;

- y de lo costoso que resulte hacerlos cumplir por algún otro medio; por ejemplo, de lo efectivo que sea el mecanismo de la reputación entre la comunidad de los negocios en un momento y lugar dados.

Cortamos aquí nuestro resumen de la elaboración teórica en torno al tamaño de la empresa, que, aunque no exhaustivo, espero que nos haya proporcionado ya suficientes hipótesis como para excitar nuestra imaginación de historiadores. Lo que hemos hecho, en efecto, es reunir un conjunto de hipótesis acerca de los determinantes del tamaño y forma concreta de la empresa, determinantes que se resumirían en costes de información, negociación y oportunismo en el mercado, y costes de control dentro de la empresa. Detrás de unos y otros, y de sus cambios a lo largo del tiempo, estarían aspectos tecnológicos y aquellas otras cosas que influyen sobre los precios relativos de los factores y de los bienes.

En estas condiciones, parece claro que una historia de la empresa que aspire a explicar algo debe consistir en la historia de los costes de información, negociación, oportunismo y control interno, y en sus determinantes tecnológicos y de otro tipo. Al tiempo que una historia así se beneficia de las hipótesis teóricas del tipo de las resumidas hasta ahora, dicha historia se convierte en el mejor banco de pruebas para la contrastación de tales hipótesis.

15 Con ello, Klein, Crawford y Alchian pueden proponer una explicación de por qué editorial e imprenta están integradas en el caso de los periódicos y, en cambio, constituyen empresas distintas en el caso de los libros. Véasc Klcin, Crawford y Alchian (1978), p. 301. 


\section{LA HISTORIA EMPRESARIAL EN UNIVERSIDADES NORTEAMERICANAS}

En centros que, como la Universidad de Harvard, ocupan las posiciones de cabeza en estudios empresariales, ése es precisamente el tipo de historia que se hace: una historia de la empresa que cxtrac sus hipótesis de la teoría de los costes de transacción. Al menos, eso es lo que hacen pensar las obras de profesionales como Alfred J. Chandler, Jr., o como Oliver Williamson.

La historia de la empresa americana es, en cualquier caso, la historia de un proceso de crecimiento, que ha culminado en la gran corporación multidivisión y multinacional. Una teoría que, como ésta, está centrada en gran parte en los determinantes del tamaño de la empresa está, pues, en las mejores condiciones para entablar una relación simbiótica con el trabajo de los historiadores cuando el objeto de estudio lo constituye la empresa USA.

Quizá el descubrimiento más sorprendente que transmiten los trabajos de Chandler y Williamson es que el crecimiento de la empresa americana ha tenido más de integración vertical que de crecimiento en horizontal. Más exactamente, la integración vertical que ahorra costes - y que, por tanto, potencialmente rebaja precios- es garantía de éxito, al contrario que la integración horizontal, que a menudo no persigue más que la elevación de esos precios. En palabras de Chandler,

"las firmas que crecieron siguiendo la vía de la integración horizontal sólo siguieron siendo rentables si, después de consolidada ésta, adoptaron una estrategia de integración vertical» 16 .

Piezas en esa explicación serían la reducción de los costes de transacción imputable a la integración vertical y, dentro de ello, la reducción de los stocks - y, por tanto, de los requerimientos de capital circulante-, así como la permanencia continuada en niveles más próximos al techo de la capacidad de la empresa. Por supuesto, la importancia de este segundo fenómeno crece en el tiempo con la importancia relativa del capital fijo, y el interés por reducir el capital circulante se redobla al desaparecer, con la mejora de los transportes, otras razones que obligaban a mantener elevados stocks. También son razones tecnológicas, en concreto el desarrollo de las comunicaciones a larga distancia, las que destruyen obstáculos a la integración empresarial, contribuyendo a su desarrollo.

Como puede verse, las innovaciones tecnológicas en la producción y distribución de bienes -o en la producción de bienes y en la producción de servicios- implican, en Chandler, alteraciones en la relación entre cos-

16 Chandler (1977), p. 315. Traducimos 
tes del mercado y costes de control dentro de la empresa, o a veces, al eliminar obstáculos de otro tipo, hacen más rentable el desarrollo de nuevos sistemas de control interno, alterando por una vía indirecta esa relación entre los costes internos y del mercado. En este sentido, el cuándo y el para qué del taylorismo, de la contabilidad de costes, del diseño de planta o del desarrollo de la estructura orgánica de la empresa son preguntas para las que Chandler tiene respuesta.

Lo impresionante de la obra cumbre de Chandler, La mano visible, es que se trata de uno de esos libros que ofrecen conjuntamente una síntesis y una detallada investigación sector por sector. Muchos de los lectores conocerán La mano visible, de forma que encontrarán innecesario un resumen, aunque sea rápido, de sus conclusiones, al tiempo que juzgarán que no hace justicia a la obra. Con los buenos libros como es éste ocurre, sin embargo, lo mismo que con los chistes buenos: que uno siente la necesidad de repetírselos a otros. Por ello, y en atención también a los lectores que no lo hayan sido - hasta ahora - de Chandler, espero que se me perdonará que haga una referencia un poco más amplia a su libro.

En los cinco capítulos - sobre un total de catorce- que La mano visible dedica íntegramente a las empresas dedicadas a la distribución de bienes se pueden encontrar detalladas descripciones de cómo el ferrocarril y el telégrafo -él mismo un producto del ferrocarril-alteraron la estrategia óptima para la obtención de información - hasta entonces consistente en la especialización en un producto y una función lo más concretos posible-, y con ello redujeron las largas cadenas comerciales existentes: a un solo intermediario donde antes había existido un mínimo de tres - comercio del algodón o venta de manufacturas a la población rural-o de cinco comercio de cereales-. Se muestra también en detalle el crecimiento de las grandes casas de venta por correo, que, apoyándose en el ferrocarril y el telégrafo, pasaron a cubrir la demanda de la población dispersa, o el desarrollo del gran almacén, esta vez fundamentado sobre la gran ciudad y la red de transporte urbano. Las impresionantes innovaciones organizativas que desarrollaron empresas como éstas, y sobre todo las que introdujeron las grandes compañías ferroviarias - la contabilidad de costes, la estructura staff-and-line y otras-, aparecen descritas, y sus razones explicadas, en este libro.

Desde luego, no todo es integración vertical en el trabajo de Chandler. Seguramente, la integración de una red ferroviaria en unas pocas grandes empresas tiene bastante más de integración vertical que de integración horizontal, pero también la intensidad en capital fijo, en energía - especialmente en calor - y el acceso a mayores mercados gracias a la revolución de los transportes abrieron el camino a economías de escala en la producción y la distribución. No obstante, la gran empresa industrial norteamericana, 
desde la siderurgia y la metalurgia no férrea hasta el tabaco, pasando por la industria de maquinaria, la química, la destilación del petróleo, la destilación de otros líquidos como los alcoholes o los refrescos de cola y, en fin, la industria de la alimentación, una vez que montaron sus grandes plantas para la fabricación acudieron a la integración vertical para reducir sus costes de transacción, a menudo impuestos por la dificultad de proveerse en el mercado de inputs en las cantidades, calidades y plazos requeridos.

Particularmente excitante es la historia de las industrias cuya producción no pudo encontrar salida a través de los distribuidores tradicionales, y que, por lo tanto, tuvieron que montar sus propias redes de distribución. Industrias que fabricaban productos nuevos - como los refrescos de cola, la leche en polvo, la carne congelada o los copos de cereales - tuvieron que volcar sus grandes recursos en la conquista directa del consumidor. Otros productos cuya distribución está sujeta a restricciones tecnológicas importantes - pensemos en la propia carne congelada, la cerveza o el petróleo-, aquellos cuya venta exige servicios especiales de demostración, instalación, atención postventa y/o crédito al comprador, como sucede con la maquinaria de todo tipo o con el material fotográfico, obligaron a las empresas que los producían a montar redes propias para la venta al cliente, agregando así las actividades de producción y distribución en otro ejemplo claro de integración vertical, esta vez hacia adelante.

En resumen, la tarea que ha llevado a cabo la mejor historia empresarial que se hace en universidades americanas es la de mostrar cómo las nuevas condiciones en cuanto a costes del mercado o costes internos de la empresa ha dado lugar a un proceso de crecimiento de la empresa americana que, sin embargo - y éste no es el lugar para tratar de las razones-, no parece haber dado lugar a verdaderos monopolios, sino más bien a oligopolios que oscilan entre la cooperación y la desconfianza mutua. El proceso se asienta, por el contrario, en una reducción de costes trasladada, al menos parcialmente, a los precios, y no en la presencia de precios de monopolio.

Más allá de lo que suceda en un sector concreto, la gran corporación americana ha diversificado sus actividades convirtiéndose en empresa multidivisión. Chandler sólo trata tangencialmente este último desarrollo, relacionando sus orígenes con las economías de escala que las empresas podían obtener extendiendo su actividad a sectores afines. Oliver Williamson, por el contrario, trata este fenómeno con mayor profundidad en varias de sus obras, manteniendo la postura de que, así como los costes de transacción en el mercado de bienes llevan a la integración vertical, los mismos costes de transacción, pero en el mercado de capitales, inducen a la gran empresa a sustituir también a este mercado en sus funciones ${ }^{17}$.

17 Véase, para una exposición resumida, Williamson (1980b), p. 187. Esta interpretación de la empresa multidivisión tiene un paralelo, para la empresa multinacional, en Jean-François Hennart (1982), (1986), (1987) y (1991). 


\section{OBRAS CITADAS}

Alchian, Armen A., y Demsetz, Harold (1972): "Production, Information Costs, and Economic Organization", American Economic Review, LXII, 5 (diciembre), pp. 777-795.

Arrow, Kenneth (1984): "The Economics of Agency", en Pratt y Zeckhauser (eds.), pp. $27-51$.

ARruñada, Benito (1990): "Control y propiedad: límites al desarrollo de la empresa espanola", Información Comercial Española, núm. 687 (noviembre), pp. 67-88.

BuCKLEY, P., y CASSON, M. (1976): The Future of Multinational Enterprise, Londres: Macmillan.

Carlos, Ana M., y Nicholas, Stephen (1990): "Agency Problems in Early Chartered Companies: The Case of the Hudson's Bay Company", Joumal of Economic History, L, 4 (diciembre), pp. 853-875.

Clarke, Roger, y MCGuinness, Tony (eds.) (1987): The Economics of the Firm, Oxford.

COASE, Ronald H. (1937): "The Nature of the Firm", Economica, núm. 386 (noviembre), pp. $386-405$.

- (1980): "La naturaleza de la empresa", Información Comercial Española, núm. 557 (enero), pp. 67.76 .

CHANDLER, Alfred J. (1977): The Visible Hand. The Managerial Revolution in American Business, Cambridge (Mass.) y Londres: The Belknap Press of Harvard University, 608 pp.

- (1980): "The United States: Seedbed of Managerial Capitalism", en Chandler y Daems (eds.), pp. 9-40.

- (1988): La mano visible, Madrid: Ministerio de Trabajo y Seguridad Social.

- (1989): Scale and Scope. The Dynamics of Industrial Capitalism, Cambridge (Mass.) y Londres: The Belknap Press of Harvard University Press, $800 \mathrm{pp}$.

CHANDLER, Alfred J., y DAEMS, Herman (eds.) (1980): Managerial Hierarcbies. Comparative Perspectives on the Rise of the Modern Industrial Enterprise, Cambridge (Mass.) y Londres: Harvard University Press, 237 pp.

DaHlman, Carl (1980): The Open Field System and Beyond: A Property Rights Study of an Economic Institution, Cambridge: Cambridge University Press.

EKELUND, Robert, y TOLLISON, Robert (1980): "Economic Regulation in Mercantile England: Heckscher Revisited", Economic Enquiry, XVIII (octubre), pp. 567-599.

- (1981): Mercantilism as a Rent Seeking Society. Economic Regulations in Historical Perspective, College Station (Texas): Texas A \& M University Press, 169 pp.

Fama, Eugene F. (1984): "Problemas de agencia y teoría de la empresa", Información Comercial Española, núm. 611 (julio), pp. 53-65.

FAMA, Eugene F., y JENSEN, Michael (1983): "Separation of Ownership and Control", Journal of Law and Economics, 25 (junio), Pp. 301-326.

FIELD, Alexander (1983): "Land Abundance, Interest/Profit Rates, and Nineteenth-Century American and British Technology", Joumal of Economic History, XLIII, pp. 405-432.

GOLDBERG, Victor (1976): "Regulation and Administered Contracts", The Bell Journal of Economics and Management Science (otoño), pp. 426-448.

GrEIF, Avner (1989): "Reputation and Coalitions in Medieval Trade: Evidence on the Maghribi Traders", Journal of Economic History, XLIV, 4 (diciembre), pp. 857-882.

HANNAH, Leslie (1976): The Rise of the Corporate Economy, Londres: Methuen.

HECKSCHER, Eli F. (1943): La época mercantilista. Historia de la organización y las ideas económicas desde el final de la Edad Media basta la sociedad liberal, México: Fondo de Cultura Económica, $871 \mathrm{pp}$.

HenNART, Jean-François (1982): A Theory of Multinational Enterprise, Ann Arbor: University of Michigan Press.

- (1986): "Internalization in Practice: Foreign Direct Investment in Malaysian Tin Mining", Joumal of Intemational Business and Economic History, XVII, 2 (verano), Pp. 131143. 
- (1987): "Transaction Costs and Multinational Enterprise: The Care of Tin", Business and Economic History, 16, pp. 147-159.

- (1991): “¿Es la teoría de la internalización una teoría general de la empresa multinacional?", Información Comercial Española, 692 (abril), pp. 133-144.

JENSEN, M. C., y MeCKLING, W. H. (1976): "Theory of the Firm: Managerial Behavior, Agency Costs and Ownership Structure", Joumal of Financial Economics, 3, pp. 305-360.

Johnson, H. T., y Kaplan, R. S. (1988): La contabilidad de costes. Auge y caída de la contabilidad de gestión, Barcelona: Plaza y Janés.

KALDOR, Nicholas (1934): "The Equilibrium of the Firm", Economic Joumal (marzo).

KleIN, Benjamin; CRAWFORD, Robert G., y AlCHIAN, Armen A. (1978): "Vertical Integration, Appropiable Rents, and the Competitive Contracting Process", Joumal of Law and Economics (octubre), pp. 297-326.

KNIGHT, Frank (1921): Risk, Uncertainty and Profit, Boston: Houghton Mifflin.

LANDES, David S. (1986): "What Do Bosses Really Do?", Joumal of Economic History, XLVI, 3 (septiembre), Pp. 585-623.

LEVY, Haim (1990): "Los costes de agencia cuando el talento del agente es desconocido: un enfoque bayesianon, Información Comercial Española, núm. 688 (diciembre), pp. 92-105.

LIPSEY, Richard G. (1967): Introducción a la economía positiva, Barcelona: Vicens Vives.

MALmgren, H. B. (1961): "Information, Expectations and the Theory of the Firm", Quarterly Joumal of Economics, LXXV, Pp. 399-421.

MCCloskey, Donald N. (1975): "The Persistence of English Common Fields", en Parker y Jones (eds.), pp. 73-119.

- (1976): "English Open Fields as a Behavior Towards Risk", Research in Economic History, I, pp. 124-170.

MCCRAW, Thomas K. (ed.) (1988): The Essential Alfred Chandler: Essays Toward a Historical Theory of Big Business, Boston (Mass.): Harvard Business School Press, 538 pp.

MCMANUS, John C. (1975): "The costs of alternative economic organizations", Canadian Journal of Economics, VIII, 3 (agosto), pp. 334-349.

NORTH, Douglas C. (1984): Estructura y cambio en la bistoria económica, Madrid: Alianza, $244 \mathrm{pp}$.

PARKER, W. N., y JONES, E. (eds.) (1975): European Peasants and Tbeir Markets, Princeton: Princeton University Press.

PENROSE, Edith T. (1980): The Growth of the Firm, Oxford.

POLLARD, Sidney (1987): La génesis de la dirección de empresa moderna. Estudio sobre la revolución industrial en Gran Bretaña, Madrid: Ministerio de Trabajo y Seguridad Social, 355 pp.

PRATT, John, y ZECKHAUSER, Richard (eds.) (1984): Principals and Agents: The Structure of Business, Boston.

ROBINSON, E. A. G. (1934): The Structure of Competitive Industry.

Ross, Stephen (1973): "The Economic Theory of Agency: The Principal's Problem", American Economic Review, 63 (mayo), pp. 134-149.

SAlas FUMÁs, Vicente (1984): "Economía teórica de la empresa", Información Comerial Española, núm. 611 (julio), pp. 9-22.

SANTOS REDONDO, Manuel (1988): "El empresario como factor de producción en el pensamiento económico de Frank H. Knight", Información Comercial Española, núm. 656 (abril), pp. $115-124$.

StLver, Morris (1983a): "Karl Polanyi and Markets in Ancient Near East: The Challenge of the Evidence", Joumal of Economic History, XLIII, pp. 795-829.

- (19836): Propbets and Markets: The Political Economy of Ancient Israel, Boston.

SMITH, Woodruff D. (1984): "The Function of Commercial Centers in the Modennization of European Capitalism: Amsterdam as an Information Exchange in the Seventeenth Century", Joumal of Economic History, XLIV, 4 (diciembre), pp. 985-1005.

Temin, Peter (1988): "Product Quality and Vertical Integration in the Early Cotton Textile Industry", Joumal of Economic History, XLVIII, 4 (diciembre), pp. 891-907. 
Universidal de CAmbridge (ed.) (1977-1982): Historia económica de Europa, Madrid: EDERSA, 7 tomos.

Williamson, Oliver E. (1971): "The Vertical Integration of Production: Market Failure Considerations", American Economic Review, 112.

- (1975): Markets and Hierarcbies: Analysis and Antitrust Implications. A Study in the economics of intemal organization, Nueva York: The Free Press, 286 pp.

- (1980a): "The Organization of Work: A Comparative Institutional Assessment", Journal of Economic Behavior and Organization, I (marzo), pp. 6-11.

- (1980b): "Emergence of the Visible Hand: Implications for Industrial Organization", en Chandler y Daems (eds.), pp. 182-202.

- (1988): The Economic Institutions of Capitalism: Firms, Markets, Relationed Contracting, Nueva York: Free Press, 450 pp.

- (1990): The Firm as a Nexus of Treaties, Londres, crc.: SAGE Publications, 358 pp.

ZORNOZA BOY, Javier (1989): "Nota sobre la evolución de los sistemas de información contable", Revista de Historia ECONÓmica, VII, 1 (invierno), pp. 197-210. 